\title{
GREEN'S FUNCTION DESCRIPTION OF SPACE-CHARGE IN INTENSE CHARGED-PARTICLE BEAMS *
}

\author{
Mark Hess, Renato Pakter, and Chiping Chen \\ MIT Plasma Science and Fusion Center, Cambridge, MA 02139
}

\section{Abstract}

We present two- and three-dimensional models of space charge in intense charged-particle beams using Green's functions. In particular, we compute the electrostatic Green's function for a periodic collinear distribution of point charges located inside of a perfectly conducting drift tube. As applications of the Green's function description, we analyze the matching and transport of an initially axisymmetric beam into a quadrupole channel and the interaction of a particle with its induced surface charge.

\section{INTRODUCTION}

Our electrostatic models of periodic space charge in an infinite conducting cylinder use both 2-D and 3-D Green's functions. The Green's function provides the complete electrostatic potential inside of the interaction region due to both point charges and induced surface charges. From this potential, the electric fields at any point inside of the cylinder can be deduced, and space charge dynamics may be simulated.

Our paper is organized as follows. In Sec. 2, both 2-D and 3-D Green's functions are obtained analytically and computed. In Sec. 3, use is made of the 2-D Green's function to simulate matching of an axisymmetric intense beam into a quadrupole focusing channel, and of the 3-D Green's function to simulate single particle dynamics.

\section{GREEN'S FUNCTION DESCRIPTION}

The 3-D Green's function satisfies Laplace's equation with periodic point charge sources [1],

$$
\nabla^{2} G_{3 D}=-\frac{4 \pi}{r} \delta\left(r-r^{\prime}\right) \delta\left(\theta-\theta^{\prime}\right) \delta_{L}\left(z-z^{\prime}\right),
$$

where

$$
\delta_{L}\left(z-z^{\prime}\right)=\sum_{n=-\infty}^{\infty} \delta\left(z-z^{\prime}-n L\right)
$$

$L$ is the spacing of the point sources, the primed coordinates denote the locations of the unit point charges. The Dirichlet boundary condition and the periodic boundary conditions for translational and rotational symmetries require that

$$
\begin{gathered}
G_{3 D}\left(r, \theta, z+L ; x^{\prime}\right)=G_{3 D}\left(r, \theta, z, x^{\prime}\right), \\
G_{3 D}\left(r, \theta+2 \pi, z ; x^{\prime}\right)=G_{3 D}\left(r, \theta, z, x^{\prime}\right), \\
G_{3 D}\left(a, \theta, z, x^{\prime}\right)=0,
\end{gathered}
$$

"Work supported by DOE and AFOSR. where $a$ is the radius of the cylinder. The solution of (1) can be constructed by expanding in terms of the eigenfunctions of Laplace's equation in cylindrical coordinates. We obtain the following solution,

$$
\begin{gathered}
G_{3 D}\left(x ; x^{\prime}\right)=\frac{2}{L} \sum_{n=-\infty}^{\infty} \sum_{l=-\infty}^{\infty} e^{i n\left(\varepsilon-\varepsilon^{\prime}\right)} e^{i l\left(\theta-\theta^{\prime}\right)} \frac{I_{l}\left(n \delta_{<}\right)}{I_{l}(n \alpha)} \\
\times\left[I_{l}(n \alpha) K_{l}\left(n \delta_{>}\right)-I_{l}\left(n \delta_{>}\right) K_{l}(n \alpha)\right],
\end{gathered}
$$

where

$$
\delta=\frac{2 \pi r}{L}, \delta^{\prime}=\frac{2 \pi r^{\prime}}{L}, \varepsilon=\frac{2 \pi z}{L}, \varepsilon^{\prime}=\frac{2 \pi z^{\prime}}{L}, \alpha=\frac{2 \pi a}{L},
$$

and the symbol $>(<)$ denote the greater (lesser) of $\delta$ and $\delta^{\prime}$. Separation of the $n=0$ terms from the $n>0$ terms yields the following form for $G_{3 D}$,

$$
\begin{aligned}
G_{3 D}=\frac{1}{L} G_{2 D} & +\frac{4}{L} \sum_{n=1}^{\infty} \cos \left[n\left(\varepsilon-\varepsilon^{\prime}\right)\right] \frac{I_{0}\left(n \delta_{<}\right)}{I_{0}(n \alpha)} \\
& \times\left\{I_{0}(n \alpha) K_{0}\left(n \delta_{>}\right)-I_{0}\left(n \delta_{>}\right) K_{0}(n \alpha)\right\} \\
+ & \frac{8}{L} \sum_{n=1}^{\infty} \sum_{l=1}^{\infty} \cos \left[n\left(\varepsilon-\varepsilon^{\prime}\right)\right] \cos \left[l\left(\theta-\theta^{\prime}\right)\right] \frac{I_{l}\left(n \delta_{<}\right)}{I_{l}(n \alpha)} \\
& \times\left\{I_{l}(n \alpha) K_{l}\left(n \delta_{>}\right)-I_{l}\left(n \delta_{>}\right) K_{l}(n \alpha)\right\},
\end{aligned}
$$

where $G_{2 D}$ is the 2D Green's function defined by,

$$
G_{2 D}=\ln \left[\frac{\alpha^{2}+\left(\delta_{>} \delta_{<} / \alpha\right)^{2}-2 \delta_{>} \delta_{<} \cos \left(\theta-\theta^{\prime}\right)}{\delta_{>}{ }^{2}+\delta_{<}{ }^{2}-2 \delta_{>} \delta_{<} \cos \left(\theta-\theta^{\prime}\right)}\right] .
$$

When we take the limit as $L \rightarrow 0$ in (6), we recover the 2-D Green's function

$$
G_{2 D}=\lim _{L \rightarrow 0} L G_{3 D}
$$

This is because the second and third terms of (6) vanish as $L \rightarrow 0$.

We tested the Green's function's convergence properties for a triply extreme case of (6) with $\delta^{\prime} \rightarrow 0$, $\alpha \rightarrow \infty$, and $\delta \rightarrow 0$. This corresponds to choosing a line charge in free space and examining the electric field between any two points. We found exact numerical agreement between the known result and the one computed from $G_{3 D}$, when we included the first 15,000 terms in the summation.

\section{APPLICATIONS}

\subsection{Two-Dimensional Case}

In many beam systems, an axisymmetric particle beam is generated at the electrode and is injected into a structure that is not axisymmetric. Typically, the rms envelope equation is used to determine the matching conditions 
for the system. However, the rms matching gives no information about the detailed phase-space evolution. Here, we make use of two-dimensional Green's function based self-consistent simulations to perform a detailed phase-space analysis for the matching and transport of a high-intensity axisymmetric particle beam in a quadrupole channel. In particular, we analyze the effects of a nonlinear transverse macroscopic velocity profile in the initial particles distribution. The nonlinearity in the velocity profile models, for instance, the effects of the concave shape of the electrode.

We consider a continuous, intense beam propagating with average axial velocity $\beta_{b} c \mathbf{e}_{\mathrm{z}}$ through a quadrupole channel with magnetic field given by

$$
\frac{q \mathbf{B}(s)}{\gamma_{b} \beta_{b} m c^{2}}=\kappa_{q}(s)\left(y \mathbf{e}_{\mathbf{x}}+x \mathbf{e}_{\mathbf{y}}\right) .
$$

Here, $q$ and $m$ are the particles charge and mass, respectively, $\gamma_{b}=\left(1-\beta_{b}^{2}\right)^{-1 / 2}$ is the relativistic mass factor, $s$ is the axial coordinate, and $\kappa_{\mathrm{q}}(s)$ is the quadrupole coupling coefficient. We assume for $\kappa_{\mathrm{q}}(s)$ the profile shown in Fig. 1, corresponding to a tapered channel where the first lattice period has quadrupoles with increasing magnetic fields that match, in an rms sense, the beam into the periodic channel formed by the remainder lattice. The lattice period is $S$.

The rms matching is obtained by analyzing the solutions of the rms envelope equations [2]

$$
\begin{aligned}
& \frac{d^{2} a}{d s^{2}}+\kappa_{q}(s) a-\frac{K}{2(a+b)}-\frac{\varepsilon^{2}}{16 a^{3}}=0, \\
& \frac{d^{2} b}{d s^{2}}-\kappa_{q}(s) b-\frac{K}{2(a+b)}-\frac{\varepsilon^{2}}{16 b^{3}}=0,
\end{aligned}
$$

where, $a \equiv\left\langle x^{2}\right\rangle^{1 / 2}$ and $b \equiv\left\langle y^{2}\right\rangle^{1 / 2}$ are the rms envelopes, $K=2 q^{2} N_{b} / \gamma_{b}^{3} \beta_{b}^{2} m c^{2}$ is the perveance of the beam, $\varepsilon$ is four times the unnormarlized rms emittance (which is assumed to be the same for $x$ and $y$ ), $N_{b}$ is the number of particles per unit axial length of beam, and \langle\rangle denotes average over particles. In particular, for a given beam intensity and periodic channel parameter $C_{3}$ (see Fig. 1), we make use of Eqs. (10) and (11) to determine the injection parameters for the axisymmetric beam, namely $a, b$ and their derivatives at $s=0$, as well as the magnetic field strengths of the quadrupoles in the first lattice period, denoted by $C_{1}$ and $C_{2}$ in Fig. 1 . We focus here on highintensity beams, which are highly space-charge dominated, such that the emittance terms in the envelope equations are negligible in comparison to the perveance terms. To illustrate the matching process, the curves in Fig. 2 show the solution for $a(s)$ and $b(s)$ for a periodic channel with vacuum phase advance $\sigma_{\mathrm{o}}=70.8^{\circ} \quad\left(S^{2} C_{3}=\right.$ 10.0, $\quad \eta=0.3$ ) and beam perveance $S K / \varepsilon=16.0$ (corresponding to a space-charge-depressed phase advance of $\sigma=5.4^{\circ}$ ).

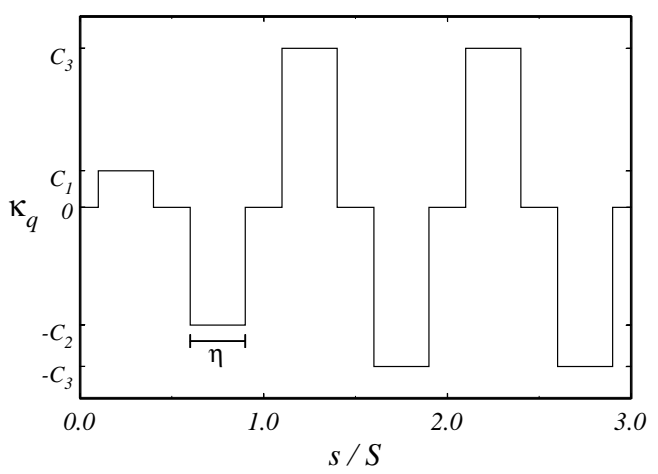

Fig. 1 Quadrupole coupling coefficient. Here, $S$ is the lattice period and $\eta$ is the filling factor.

To analyze the effects of the nonlinear tranverse macroscopic velocity profile in the phase-space evolution of the beam we make use of self-consistent particle simulation. We consider an initially axisymmetric cold beam with particles homogeneously distributed up to a radius of $r_{b}=2 a(0)=2 b(0)$ and with transverse velocity given by

$$
\mathbf{r}^{\prime}=\frac{a^{\prime}}{a} \mathbf{r}\left[1+v\left(1-\frac{\mathbf{r}^{2}}{2 a^{2}}\right)\right],
$$

where $\mathbf{r}=x \mathbf{e}_{\mathrm{x}}+y \mathbf{e}_{\mathrm{y}}$ is the transverse displacement, the prime denotes derivative with respect to $s$, and $v$ is the parameter that measures the nonlinearity in the velocity profile (for $v=0$ the velocity profile is linear). It can be shown that the nonlinearity induced by the concave shape of the electrode leads to $v>0$.

First, we analyze the behavior of the rms envelopes. The symbols in Fig. 2 correspond to the rms envelopes obtained from the particle simulation for an initial velocity nonlinearity of $v=0.25$. The channel and beam parameters are the same as those used in the rms envelope equations represented in the figure by the curves. The evident agreement reveals that even in the presence of nonlinearities in the velocity profile, the beam matches well (in an rms sense) to the quadrupole channel.

Second, we analyze what happens to the particle distribution as the nonlinearity in the velocity profile is introduced. The results are summarized in Fig. 3, where we compare particle distributions for the cases with and without initial velocity nonlinearities at three axial positions: $s / S=0.0,1.0$ and 2.5. Note that at these axial positions the beam is such that $a(s)=b(s)$. In Fig. 3, the plots shown on the left correspond to $v=0$ and those on the right correspond to $v=0.25$. While for $v=0$ the beam propagates coherently, for $v=0.25$ the initially rounded beam develops edges after the first lattice period, becoming partially hollow subsequently (at $s / S=$ 
2.5). The hollow profile of the beam at $s / S=2.5$ is confirmed by analyzing the density function (not presented here), which shows that the density at the edge is twice the density at the center of the beam.

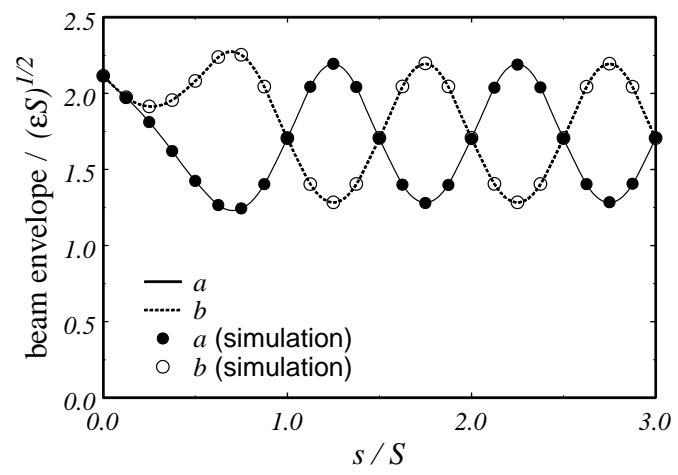

Fig. 2 RMS envelopes obtained from the rms envelope equations and self-consistent simulation.
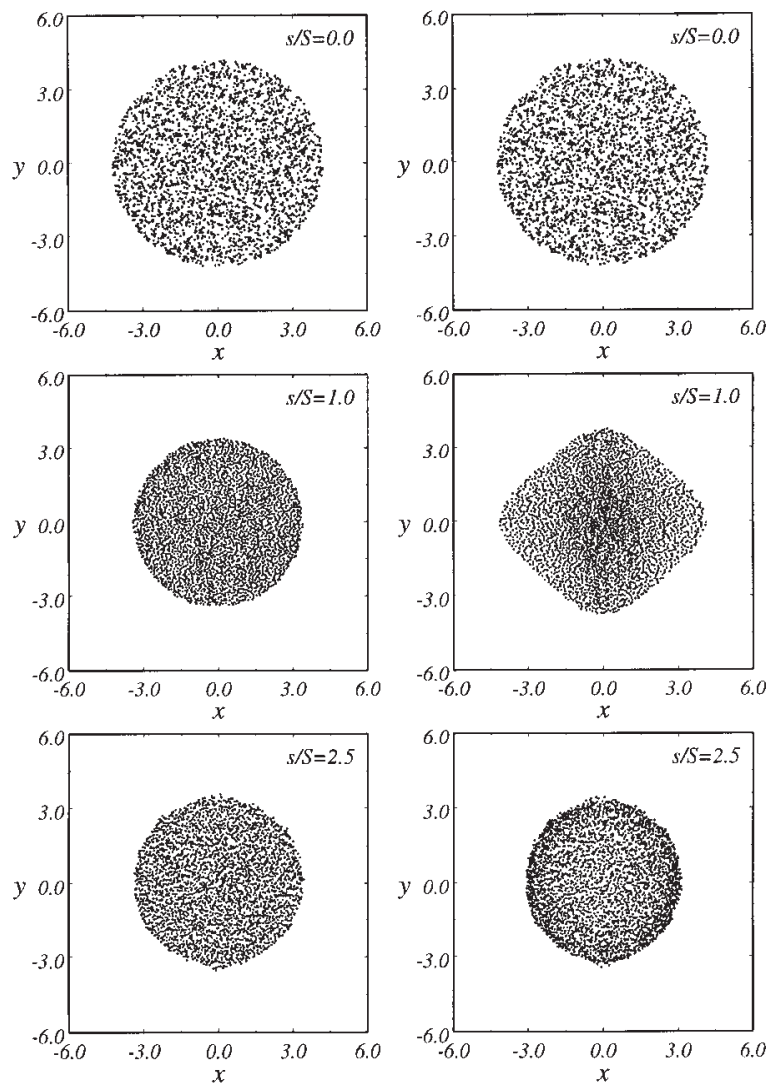

Fig. 3 Particles distribution for $v=0$ (left) and $v=0.25$ (right). The coordinates $x$ and $y$ are normalized to $\sqrt{\varepsilon S}$.

To summarize briefly, we have analyzed the matching and transport of an initially axisymmetric highintensity beam in a tapered quadrupole channel. The rms envelope equations have been used to determine the matching conditions. Using a two-dimensional Green's function based self-consistent simulation code, we have investigated the effects of an initial nonlinear transverse macroscopic velocity profile on the beam dynamics. It has been found that the rms radii evolution agrees with the rms envelope equation analysis, and that the presence of nonlinearities in the velocity leads to changes in the detailed particles distribution and the onset of beam hollowing.

\subsection{Three-Dimensional Case}

As a necessary component of a one-particle simulation, we compute the electrostatic self-field (field of a particle due to its induced surface charge. This radial electric field is given by the following expression:

$$
\begin{aligned}
E_{\text {self }}= & \frac{4 \pi}{L^{2}}\left(\frac{\delta}{\alpha^{2}-\delta^{2}}\right)+\frac{8 \pi}{L^{2}} \sum_{n=1}^{\infty} \frac{n I_{0}(n \delta) I_{1}(n \delta) K_{0}(n \alpha)}{I_{0}(n \alpha)} \\
& +\frac{16 \pi}{L^{2}} \sum_{n=1}^{\infty} \sum_{l=1}^{\infty} \frac{n I_{l}(n \delta) I_{l}^{\prime}(n \delta) K_{l}(n \alpha)}{I_{l}(n \alpha)}
\end{aligned}
$$

Utilizing the electrostatic self-field force, we are able to simulate one string of particles interacting with its induced charge. We apply a constant magnetic field parallel to the axis of the pipe. The Lorentz forces are only in the transverse direction, so longitudinal motion can be ignored. As an example, a radially confined orbit is shown in Fig. 4 for $\alpha=2 \delta$ (initial) $=0.2$.

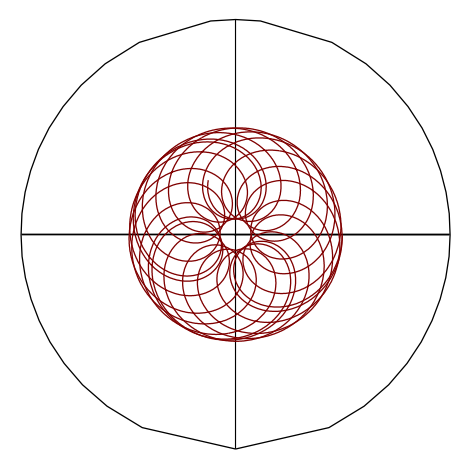

Fig. 4 Trajectory of a trapped particle in the conducting pipe with initial conditions $\mathrm{P}_{\mathrm{r}}=\theta=0, \quad \mathrm{P}_{\theta}=0.001$ (normalized to $8 \pi^{2} c / q L^{2} B$ ), and $\xi=32 \pi^{2} m c^{2} / L^{3} B^{2}=0.001$.

\section{SUMMARY}

Both two- and three-dimensional models of space charge in intense charged-particle beams have been presented using Green's functions, and applied, respectively, in the analyses of the matching and transport of an initially axisymmetric beam into a quadrupole channel and of the interaction of a particle with its induced surface charge.

\section{REFERENCES}

[1] J. D. Jackson, Classical Electrodynamics (John Wiley, New York, 1975).

[2] F. J. Sacherer, IEEE Trans. Nucl. Sci. NS-18, 1105 (1971). 